\title{
Identification of mundoserone by zebrafish in vivo screening as a natural product with anti-angiogenic activity
}

\author{
KAN CHEN, CHANGQIAN WANG, YUQI FAN, JUN GU, ZHIHUA HAN, \\ YUE WANG, LIN GAO and HUASU ZENG \\ Department of Cardiology, Shanghai Ninth People's Hospital \\ Affiliated to Shanghai Jiaotong University School of Medicine, Shanghai 200011, P.R. China
}

Received November 30, 2017; Accepted June 15, 2018

DOI: $10.3892 /$ etm.2018.6748

\begin{abstract}
The present study aimed to screen natural products with anti-angiogenic potential from the Natural Products Collection of MicroSource. The anti-angiogenic activity of 240 natural products was assessed using the zebrafish line $\mathrm{Tg}\left(\mathrm{fli1}\right.$ a: EGFP) ${ }^{\mathrm{y} 1}$. At $24 \mathrm{~h}$ post-fertilization, the embryos were treated with the library compounds for $24 \mathrm{~h}$ and, the morphology of the intersegmental vessels (ISVs) was then assessed using a fluorescence microscope, followed by counting of ISVs and calculation of the inhibition ratio. The expression of angiogenesis-associated genes was determined by quantitative polymerase chain reaction. The results indicated that mundoserone inhibited ISV formation in zebrafish embryos in a dose-dependent manner, with a significant anti-angiogenic activity observed at a concentration of $10 \mu \mathrm{M}$, leading to an ISV inhibition ratio of $73.6 \pm 1.3 \%$. Mundoserone significantly reduced the expression of slit guidance ligand 3 (SLIT3), roundabout guidance receptor 1 (ROBO1) and -2, fibroblast growth factor receptor (FGFR)2 and -3, as well as protein tyrosine phosphatase, receptor type B (PTP-RB), but increased the expression of NOTCH1A. Accordingly, mundoserone may be an effective angiogenic inhibitor, which acts via downregulation of SLIT/ROBO1 and FGFR/PTP-RB, and upregulation of NOTCH1A signaling.
\end{abstract}

Correspondence to: Dr Changqian Wang or Dr Yuqi Fan, Department of Cardiology, Shanghai Ninth People's Hospital Affiliated to Shanghai Jiaotong University School of Medicine, 639 Zhi Zaoju Road, Shanghai 200011, P.R. China

E-mail: changqianwang17@hotmail.com

E-mail: yuqifan17@hotmail.com

Abbreviations: ISVs, intersegmental vessels; VEGF, vascular endothelial growth factor; FGF, fibroblast growth factor; NPs, natural products; EGFP, enhanced green fluorescent protein; hpf, hours post-fertilization; RT-qPCR, reverse transcription-quantitative polymerase chain reaction

Key words: angiogenesis, inhibitor, natural products, mundoserone, slit guidance ligand 3/roundabout guidance receptor, fibroblast growth factor

\section{Introduction}

Angiogenesis is defined as the formation of new blood vessels from the pre-existing vascular system through endothelial proliferation and migration due to abnormal expression of several pro-angiogenic factors, including vascular endothelial growth factor (VEGF), fibroblast growth factor (FGF), their receptors and genes of associated signaling pathways (e.g., phosphoinositide-3 kinase/Akt, mitogen-activated protein kinase and Notch) (1-4). Accumulating evidence suggests that angiogenesis is an essential process for the initiation and development of numerous diseases, including cancer (5), psoriasis (6), rheumatoid arthritis (7), retinopathy (8) and endometriosis (9). Therefore, targeted inhibition of angiogenesis may potentially be beneficial for the treatment of the above diseases, which has been demonstrated in clinical trials on VEGF inhibitors (e.g. dovitinib, sunitinib, sorafenib or bevacizumab) $(10,11)$. However, high proportional rates of adverse events caused by the currently available drugs limits their wide application and acceptability $(10,11)$. The aforementioned experimental results indicate the requirement of more effective and safe angiogenesis inhibitors.

Natural products (NPs) are biologically active secondary metabolites produced by plants, animals and microorganisms. NPs have long served as an important source for drug discovery due to their potentially low toxicity. It is estimated that $>2 / 3$ of Food and Drug Administration-approved drugs are NPs or their derivatives (12). Thus, screening of NPs has been proposed as an important approach to obtain effective and safe angiogenesis inhibitors. Although previous studies have reported the anti-angiogenic effects of certain NPs, including genistein (13), camptothecin (14), kaempferol (15), ferulic acid (16) and quercetin (17), studies that identify anti-angiogenic NPs by screening of a drug library remain rare.

The goal of the present study was to screen NPs with anti-angiogenic activity from the Natural Products Collection of MicroSource Discovery Systems (http://www.msdiscovery. com) (18) using the zebrafish embryo in vivo model. This model allows for direct visualization of the vascular system via endothelium-specific enhanced green fluorescent protein (EGFP) expression in the $\mathrm{Tg}(\text { flila: EGFP) })^{\mathrm{y} 1}$ zebrafish line, which ensures rapid evaluation of the responses of live embryos to drugs (19). In the present study, mundoserone 
was identified to have potent anti-angiogenic activity in zebrafish embryos and this effect was dose-dependent. The present results may provide a theoretical basis for the future clinical use of this compound to treat diseases associated with excessive angiogenesis.

\section{Materials and methods}

Zebrafish care and maintenance. The zebrafish line $\operatorname{Tg}$ (flila: EGFP ${ }^{\mathrm{y} 1}$ with transgenic endothelial cells expressing EGFP was provided by the Shanghai Research Center for Model Organisms (Shanghai, China). The zebrafish were maintained in a constant flow water system at a temperature of $28.5^{\circ} \mathrm{C}$ under a 14-h light/10-h dark cycle. All protocols were in accordance with the guidelines of the American Association for Accreditation of Laboratory Animal Care (20) and approved by the Institutional Animal Care and Use Committee of Shanghai Ninth People's Hospital (Shanghai, China).

Embryo collection and drug treatment. Zebrafish embryos were generated by natural pair-wise mating (5-6 pairs for the generation of 200-300 embryos) and raised at $28.5^{\circ} \mathrm{C}$ in deionized water. At $24 \mathrm{~h}$ post-fertilization (hpf), embryos were distributed into 12-well plates (30 embryos/well) in 0.2\% Instant Ocean Salt (Aquarium Systems, Inc., Mentor, OH, USA) in deionized water and treated with $5 \mu \mathrm{M}$ PTK787 [a VEGF receptor (VEGFR) antagonist for generation of a positive control group; Selleck Chemicals, Houston, TX, USA; dissolved in $0.4 \% \mathrm{v} / \mathrm{v}$ dimethyl sulfoxide (DMSO)], 0.1\% DMSO (negative control) and $10 \mu \mathrm{M}$ natural product (Natural Products Collection, Microsource, Gaylordsville, CT, USA) for $24 \mathrm{~h}$ to screen anti-angiogenic drugs (Fig. 1), of which mundoserone was selected. Subsequently, other embryos were added to 12-well plates (30 embryos per well) and exposed to $2.5,5$ and $10 \mu \mathrm{M}$ of mundoserone for $24 \mathrm{~h}$ to observe the concentration-dependent anti-angiogenic effects of mundoserone. All of the experiments were repeated three times.

Angiogenesis assessment. After $48 \mathrm{hpf}, 10$ embryos in each well were anesthetized with $0.016 \%$ MS-222 (tricaine methanesulfonate; Sigma-Aldrich; Merck KGaA, Darmstadt, Germany) and the morphology of the intersegmental vessels (ISVs), which normally connect the dorsal longitudinal anastomotic vessels (DLAVs) to the dorsal aorta (DA), was visually assessed using a Nikon SMZ 1500 Fluorescence microscope (Nikon Corp., Tokyo, Japan) equipped with a digital camera. Quantitative image analyses were performed using image based morphometric analysis software (NIS-Elements D3.1; Nikon Corp.) and Adobe Photoshop 7.0 software (Adobe Systems, Inc., San Jose, CA, USA). A total of 10 embryos per group were evaluated by two blinded observers in 3 independent experiments. Drug effects were calculated according to the following formula: $\%$ Inhibition $=\left(1-\mathrm{ISV}_{\text {concentration of compound }} / \mathrm{ISV}_{\text {vehicle }}\right) \times 100 \%$.

Reverse transcription-quantitative polymerase chain reaction $(R T-q P C R)$. The effects of mundoserone on the expression of angiogenesis-associated genes were determined by RT-qPCR. Total RNA was extracted from 30 embryos that had been treated with DMSO or $10 \mu \mathrm{M}$ mundoserone for
24 h using TRIzol reagent (cat. no. 15596026; Invitrogen; Thermo Fisher Scientific, Inc., Waltham, MA, USA). RNA was reverse transcribed using the PrimeScript RT reagent kit with gDNA Eraser (cat. no. RR047A; Takara Bio, Inc., Otsu, Japan). Quantification of the gene expression was performed in triplicate using Bio-Rad iQ SYBR Green Supermix (Bio-Rad Laboratories, Inc., Hercules, CA, USA) with detection on the Realplex system (Eppendorf, Hamburg, Germany). Gene expression was relatively quantified based on the comparative threshold cycle method $\left(2^{-\Delta \Delta \mathrm{Cq}}\right)$ (21) using $\beta$-actin as an endogenous control gene. Primer sequences are listed in Table I.

Statistical analysis. Values are expressed as the mean \pm standard error of the mean. Statistical analysis and graphical representation of the data were performed using GraphPad Prism software (version 5.0; GraphPad Software, Inc., La Jolla, CA, USA). Statistical significance of differences in the number of ISVs among the negative control and positive control groups as well as those treated with different doses of mundoserone was assessed using analysis of variance followed by Tukey's post-hoc test for multiple comparisons. The difference in gene expression between the control and mundoserone groups was analyzed using Student's t-test. $\mathrm{P}<0.05$ was considered to indicate a statistically significant difference.

\section{Results}

Mundoserone inhibits the formation of the ISVs in zebrafish. Transgenic zebrafish with fluorescent blood vessels were used to screen angiogenic inhibitors from 240 compounds commercially available from the Microsource NP library. Of the compounds in the library, mundoserone, was identified to have anti-angiogenic activity, for which it has not been previously reported, to the best of our knowledge. Exposure of zebrafish to mundoserone inhibited the development of the ISVs and DLAV in comparison with the vehicle and positive control (PTK787) (Fig. 1).

Furthermore, the dose-response effect of mundoserone on the ISVs of zebrafish was also investigated. As presented in Fig. 2, the ISVs were generally intact, but appeared thinner in zebrafish treated with $2.5 \mu \mathrm{M}$ mundoserone for $24 \mathrm{~h}$. In addition to becoming thinner, certain ISVs were incompletely formed in zebrafish treated with $5 \mu \mathrm{M}$ mundoserone for $24 \mathrm{~h}$. Of note, most of the ISVs in zebrafish were incomplete and DLAV development was inhibited by $10 \mu \mathrm{M}$ mundoserone at $48 \mathrm{hpf}$. These results suggested that the anti-angiogenic effect of mundoserone was dose-dependent, which was further indicated by the quantitative results: The number of ISVs remaining in zebrafish following exposure to $5(21.4 \pm 0.5)$ or $10 \mu \mathrm{M}(7.3 \pm 1.2)$ mundoserone was significantly lower compared with the negative control (27.6 \pm 0.5$)$. Similarly, the ISV inhibition ratio was higher in zebrafish following exposure to $10 \mu \mathrm{M}$ mundoserone $(73.6 \pm 1.3 \%)$ compared with the control $(0 \pm 0.6 \%), 2.5(0 \pm 0.6 \%)$ or $5 \mu \mathrm{M}(22.5 \pm 0.6 \%)$ mundoserone groups.

Mundoserone exerts anti-angiogenesis effects via the downregulation of slit guidance ligand 3 (SLIT3)/roundabout guidance receptor $(R O B O) 1$ and $F G F$ receptor $(F G F R) /$ protein tyrosine phosphatase, receptor type $B(P T P-R B)$, and the upregulation of NOTCHIA. To investigate the potential 
Table I. Primer sequences.

\begin{tabular}{lll} 
Gene & \multicolumn{1}{c}{ Forward (5'-3') } & \multicolumn{1}{c}{ Reverse (5'-3') } \\
\hline DLL4 & AGGCCTGGCACTCACCTTACTC & CACCCCAGCCCTCTTTACAGTT \\
NOTCH1A & GCCGCAGATGCAGGGCAATGAAGT & GAGGGCAGGCAGGGCTGGTAGAGG \\
HEY2 & CGGCTTCCGGGAGTGTCTGACT & TCCCCACGGTCGGTATGGTTA \\
EFNB2A & TTGGGGCTGGAGTTCTTCAGA & TCTTGGGCGTGGCTAATGTGCT \\
SLIT2 & GAGCGACTGGACCTGAATG & GTAGATCCTGAAATGCCCCTC \\
SLIT3 & GCGAGTGTTTCCAAGACCTG & GATTTCATTGTCGTTCAGCCG \\
ROBO1 & AGGAGTCACATACAGGCTAGAG & GTCTGAGATCTGCTGGGAAATG \\
ROBO2 & GAGGTGTGGATGTGGACTATG & CTACAATCCGAGGTGGAGAATC \\
ROBO4 & GAGATCAGTCCCAAACCACAG & CCCACAGATATAGCCCAACG \\
FGFR2 & CCCCGACAACCGCACGCTCGTA & TAGCCGCCCATGCGATCCTCCTGT \\
FGFR3 & TCCCCGTATCCAGGTATCC & TCTGAACGTGGGTCTTTGTG \\
COX2 & CCTTCCGGCCATCATTCTTATT & CCGCAGATTTCAGAGCATTGTC \\
PTP-RB & TTGGGCAGCATGCGGAATACTGAG & TTACCAGGCTGCCATGAAACATCC \\
VEGFAA & CTCCATCTGTCTGCTGTAAAGG & GGGATACTCCTGGATGATGTCTA \\
VEGFR-2 & CCTGAGACCATCTTTGACCG & GTTCCCTCTTTAAGTCGCCTG \\
VEGFR-1 & GTATTTGAACAGCACGGGTTTAG & CGGCTTCTTGATATGCGTTTG \\
PIK3R2 & CCCGGAAACTGCTCCCCCTAATCT & AGCGGGAGGAGTCGGCTCTTGTT \\
$\beta$-actin & TCCGGTATGTGCAAAGCCGG & CCACATCTGCTGGAAGGTGG
\end{tabular}

FGFR, fibroblast growth factor receptor; SLIT3, slit guidance ligand 3; ROBO, roundabout guidance receptor; PTP-RB, protein tyrosine phosphatase, receptor type B; DLL, deltalike ligand 4; COX, cyclooxygenase; VEGFR, vascular endothelial growth factor receptor; PIK3R2, phosphoinositide-3-kinase regulatory subunit 2; HEY2, hes-related family basic helix-loop-helix transcription factor with YRPW motif 2; EFNB2, ephrin B2.
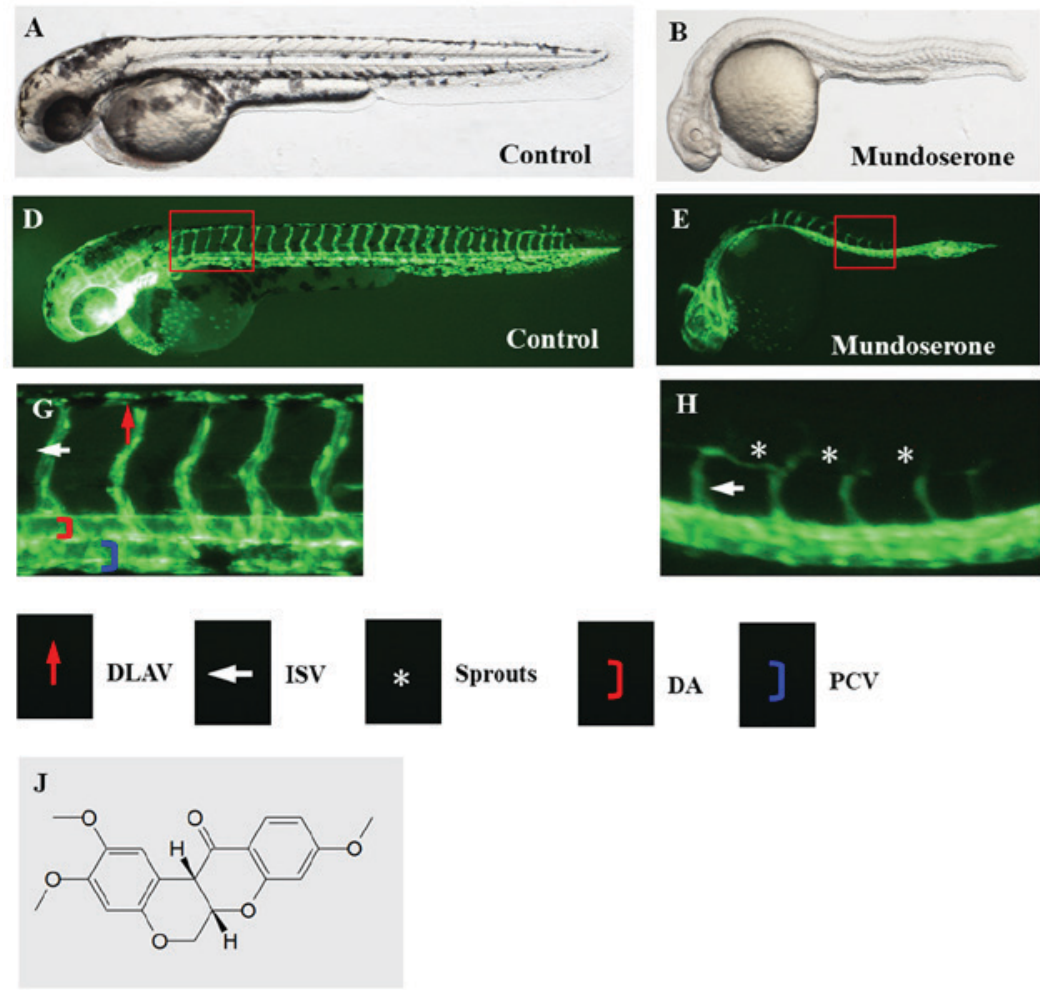
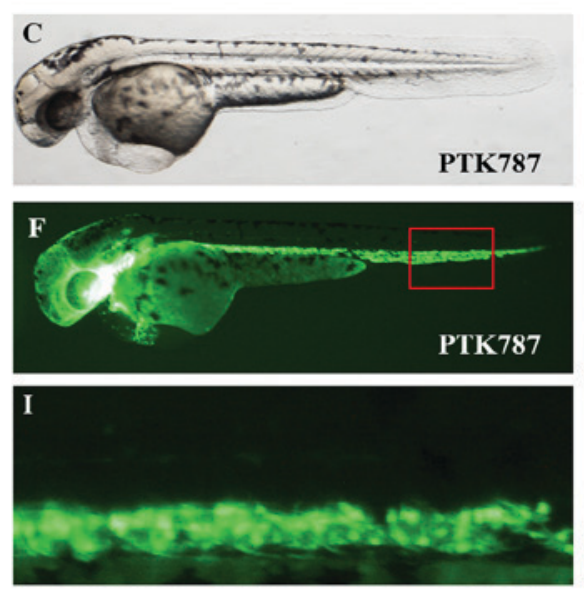

Figure 1. Among the natural products screened in Tg(flila: EGFP) ${ }^{\mathrm{y} 1}$ zebrafish, mundoserone was identified as an anti-angiogenic compound. Representative (A-C) bright-field and (D-F) fluorescent images of zebrafish embryos at $24 \mathrm{~h}$ post-fertilization treated with $0.1 \%$ dimethyl sulfoxide (control), $10 \mu \mathrm{M}$ mundoserone or $5 \mu \mathrm{M}$ PTK787 (positive control) for $24 \mathrm{~h}$ (magnification, x40). (G-I) Magnification of images (D-F) (magnification, x112.5). Compared with those in the control group, embryos treated with mundoserone presented with a lower number of incomplete ISVs and only occasional sprouts (asterisks) of the DA were observed. (J) Chemical structure of mundoserone. DLAV, dorsal longitudinal anastomotic vessels; ISVs, intersegmental vessels; DA, dorsal aorta; PCV, posterior cardinal vein. 

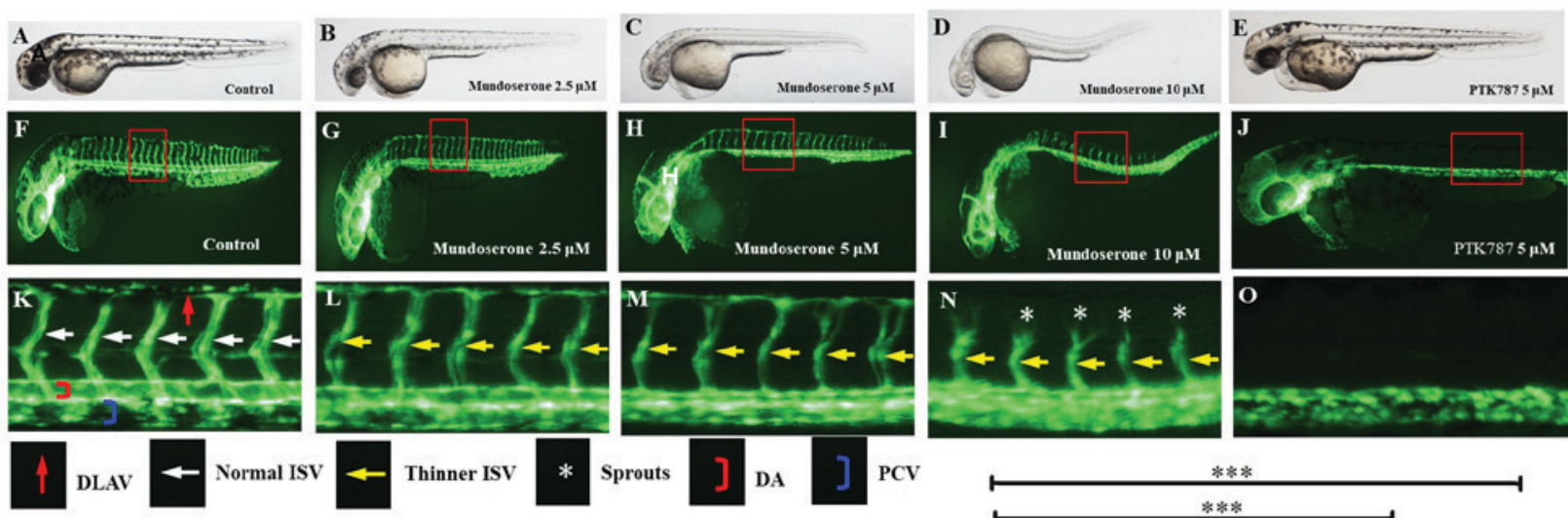

P

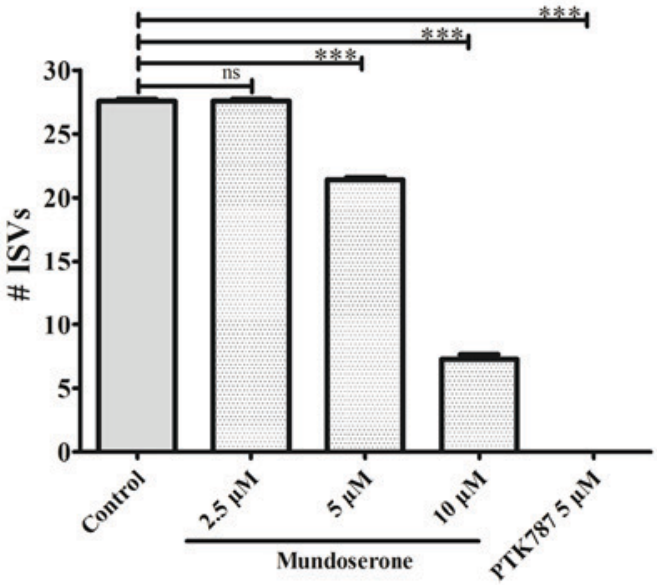

Q

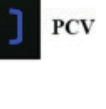

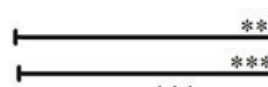

$* * *$
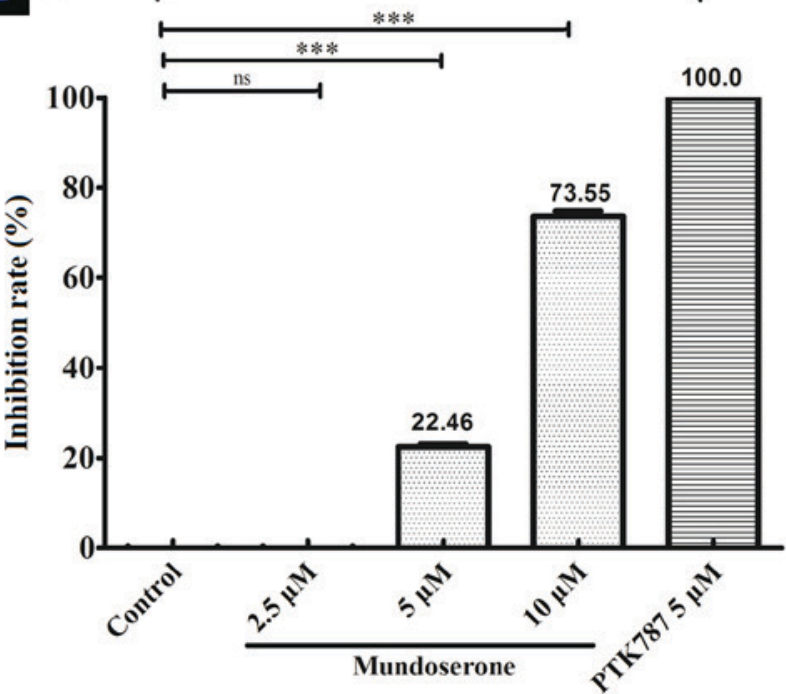

Figure 2. Mundoserone inhibits the trunk angiogenesis of zebrafish in a dose-dependent manner. Representative (A-E) bright field and (F-J) fluorescent images of zebrafish embryos at $24 \mathrm{~h}$ post-fertilization treated with $0.1 \%$ dimethyl sulfoxide (control), mundoserone $(2.5,5$ or $10 \mu \mathrm{M})$ or $5 \mu \mathrm{M}$ PTK 787 (positive control) for $24 \mathrm{~h}$ (magnification, x40). (K-O) Magnification of images F-J (magnification, x112.5). Compared with the control, embryos exposed to mundoserone exhibited a lower number of incomplete ISVs and only occasional sprouts (asterisks) of the DA were observed. Quantification of (P) the number of complete ISVs and (Q) inhibition rate in mundoserone-treated embryos. Values are expressed as the mean \pm standard error of the mean $(n=10)$. ${ }^{* * *} \mathrm{P}<0.001$. $n s$, not significant; DLAV, dorsal longitudinal anastomotic vessels; ISVs, intersegmental vessels; DA, dorsal aorta; PCV, posterior cardinal vein.

mechanisms of the anti-angiogenesis effects of mundoserone, RT-qPCR was performed to analyze the expression of genes involved in angiogenesis-associated signaling pathways in embryos treated with $10 \mu \mathrm{M}$ mundoserone. The results indicated that mundoserone significantly reduced the expression of SLIT3, ROBO1, ROBO2, FGFR2, FGFR3 and PTP-RB, but increased the expression of NOTCH1A. No significant difference was observed in the expression of cyclooxygenase 2, SLIT2, ROBO4, deltalike ligand 4 (DLL4), hes-related family basic helix-loop-helix transcription factor with YRPW motif 2 and ephrin B2A between the mundoserone treatment and negative control groups (Fig. 3). As an unexpected result, mundoserone promoted the expression of VEGFAA, VEGFR-2 and VEGFR-1.

\section{Discussion}

To the best of our knowledge, the present study was the first to identify mundoserone as a potential anti-angiogenic drug, which dose-dependently suppressed the formation of ISVs in a zebrafish embryo model. This inhibitory effect may be exerted by blocking of the SLIT/ROBO and FGFR pathways, as well as activation of NOTCH1A signaling.
Although studies investigating the role of mundoserone are rare (22), mundoserone is a structural analog of rotenone, and extensive studies have demonstrated that rotenone inhibits cell proliferation and migration. For instance, Srivastava and Panda (23) reported that rotenone inhibited the proliferation of HeLa and MCF-7 cells by perturbing microtubule assembly dynamics, with half maximal inhibitory concentrations of $0.2 \pm 0.1$ and $0.4 \pm 0.1 \mu \mathrm{M}$, respectively. Ishido and Suzuki (24) indicated that exposure to rotenone inhibited the migration, decreased the proliferation and increased the apoptotic rate of mesencephalic neural stem cells in a dose-dependent manner. In addition, administration of rotenone for $6 \mathrm{~h}$ was reported to result in a significant dissipation of the mitochondrial membrane potential in microvascular endothelial cells, indicating the disruption of the mitochondrial respiratory chain and induction of apoptosis (25). These results imply that application of mundoserone or rotenone may possibly prevent excessive angiogenesis, and this hypothesis was also demonstrated in the present study, as a $73.55 \%$ ISV inhibition ratio was achieved with mundoserone.

Although VEGFs and FGF are considered as indispensable angiogenic factors for angiogenesis (1-4), the results of the present study suggested that mundoserone did not exert any anti-angiogenic activity by inhibiting VEGFs and VEGFRs, 

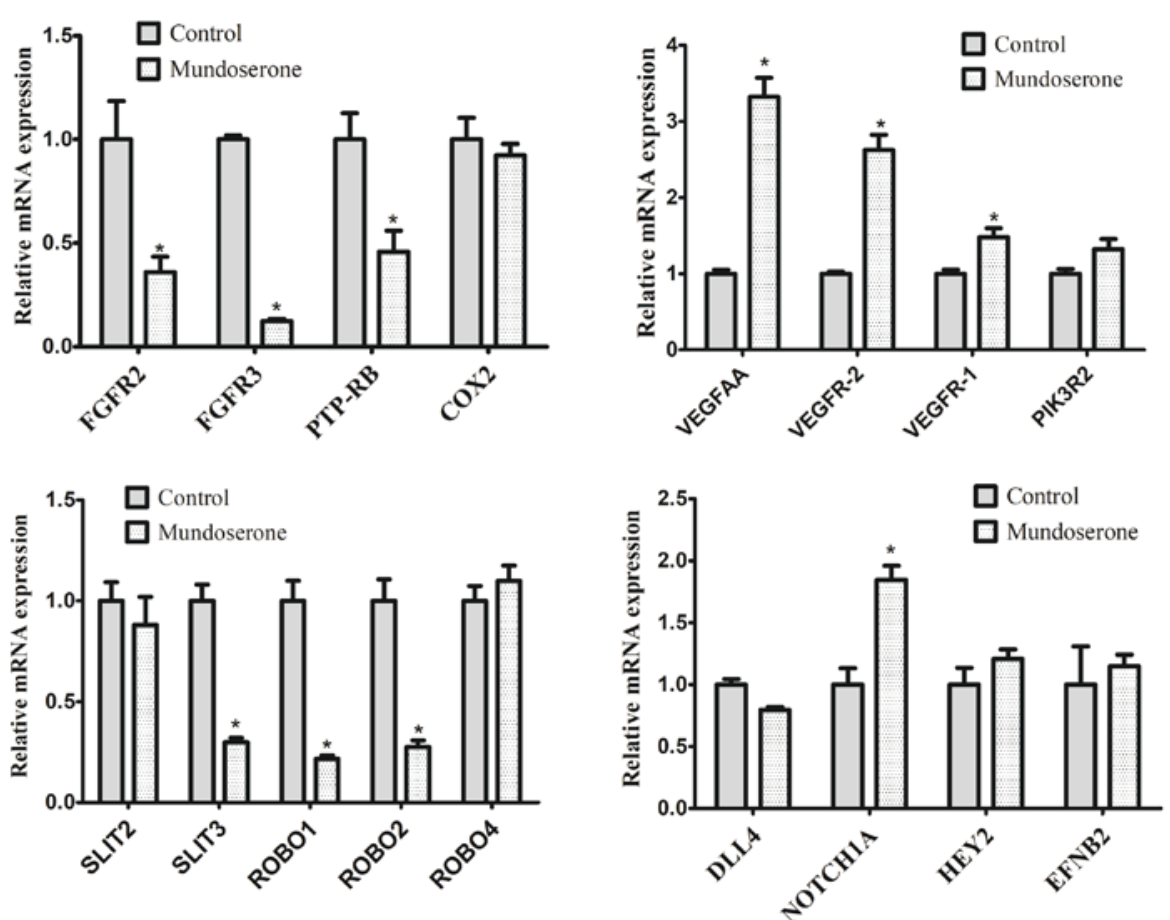

Figure 3. Expression of angiogenesis-associated genes in zebrafish embryos after treatment with $10 \mu \mathrm{M}$ mundoserone. Values are expressed as the mean \pm standard error of the mean $(n=4)$. "P<0.05 vs. Control. FGFR, fibroblast growth factor receptor; SLIT3, slit guidance ligand 3; ROBO, roundabout guidance receptor; PTP-RB, protein tyrosine phosphatase, receptor type B; DLL, deltalike ligand 4; COX, cyclooxygenase; VEGFR, vascular endothelial growth factor receptor; PIK3R2, phosphoinositide-3-kinase regulatory subunit 2; HEY2, hes related family bHLH transcription factor with YRPW motif 2; EFNB2, ephrin B2.

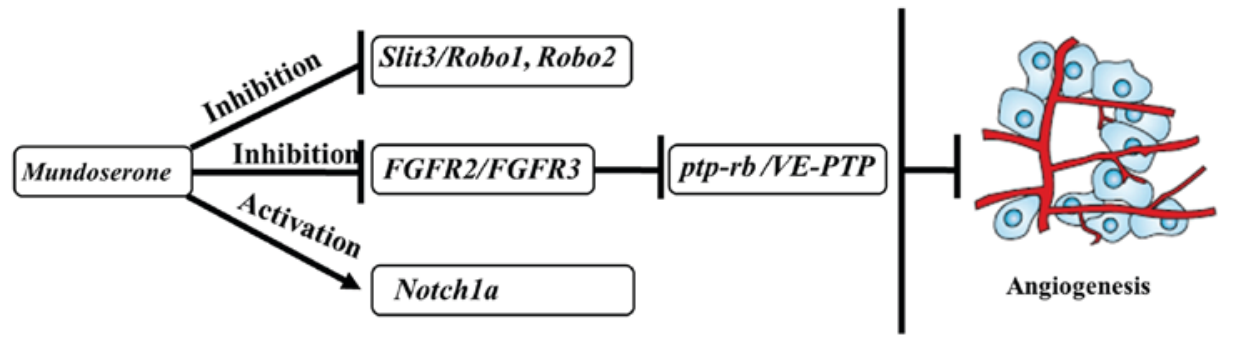

Figure 4. Schematic model illustrating the mechanism of action of mundoserone on angiogenesis. FGFR, fibroblast growth factor receptor; SLIT3, slit guidance ligand 3; ROBO, roundabout guidance receptor; PTP-RB, protein tyrosine phosphatase, receptor type B; VE-PTP, vascular endothelial cell-specific phosphotyrosine phosphatase.

but only FGFR2 and -3 . This may be a potential reason of the failure of blockage of angiogenesis by only using VEGF inhibitors in certain clinical patients. VEGF-independent angiogenic pathways should be considered when resistance to VEGF-targeted therapies is encountered (16). Furthermore, in vitro studies demonstrated that suppression of FGF signaling reduced the expression levels of PTP non-receptor type 11 (PTPN11) and then disrupted the PTPN11/VE-cadherin interaction, leading to the loss of endothelial junction integrity (26) and terminating endothelial cell interface elongation, ultimately preventing angiogenesis (27). As expected, PTP-RB expression was also lower after mundoserone treatment, indirectly inferring that mundoserone inhibits angiogenesis via the FGFR2/FGFR3/PTP-RB interaction.

In addition to VEGFs and FGF, SLIT/ROBO signaling has also been reported to have a significant role in angiogenesis $(28,29)$. SLIT2 stimulation induced the expression of ROBO1 in lymphatic endothelial cells and mediation of the migration and tube formation, which was reversed by using the SLIT/ROBO-specific antibodies (30). Further in vivo experiments confirmed that overexpression of SLIT2 significantly enhanced tumor lymphangiogenesis and subsequently promoted mesenteric lymph node metastasis of pancreatic islet tumors (30). The mRNA and protein levels of SLIT3, ROBO1 and ROBB4 were reported to be significantly increased in human umbilical vein endothelial cells after hypoxia, which is a common cause for preeclampsia (31). SLIT2 was demonstrated to induce ocular neovascular diseases by promoting sprouting angiogenesis in retinas through ROBO1 and ROBO2 (32). Thus, blockade of SLIT/ROBO signaling may be therapeutically exploited to inhibit angiogenesis. As anticipated, mundoserone significantly reduced the expression of SLIT3, as well as ROBO1 and -2 in zebrafish embryos in the present study.

Although the role is controversial, studies have suggested that activation of the Notch signaling pathway may be a potential approach of inhibiting angiogenesis and preventing tumor 
metastasis $(33,34)$. For instance, Banerjee et al (33) reported that inhibition of NOTCH via a soluble NOTCH1 decoy, which acts as antagonists of DLL and protein jagged ligands, caused a marked increase in liver metastasis of neuroblastoma and breast cancer. This result was also confirmed in transgenic mice with heterozygous loss of NOTCH1 (33). A study by Lee et al (34) further illustrated that NOTCH activation suppresses retinal angiogenesis by reducing the expression of transcription factor sex-determining region $\mathrm{Y}$ box 17 , which may be inhibited by DLL4 blockade. By constructing a transgenic mouse model with Cre-conditional expression of the constitutively active intracellular domain of NOTCH1, Liu et al (35) demonstrated that NOTCH signaling may retard basic FGF-induced angiogenesis and ovarian follicle development. In line with these studies, the present results also suggested that mundoserone treatment increased the expression of NOTCH1A and thus inhibited angiogenesis in zebrafish.

Of note, the present study had certain limitations. First, the study only preliminarily screened mundoserone as a natural product with anti-angiogenic activity using a zebrafish embryo model. The efficiency of mundoserone in inhibiting angiogenesis in humans and the optimal concentration require further validation in a series of mammalian models (i.e., mice, monkey) and phase-III clinical trials. Second, further in vitro studies are necessary to confirm the effects of mundoserone on the proliferation and migration of microvascular endothelial cells and the expression of associated pathway proteins (SLIT, ROBO1, FGFR, PTP-RB and NOTCH1A) $(15,16)$. Third, the safety of mundoserone should be further evaluated by in vivo and in vitro experiments in the future because growth retardation of the embryos was observed with mundoserone treatment. Fourth, the VEGF-independent, but FGF-dependent mechanisms of mundoserone should also be validated. In addition, the unexpected upregulation of VEGFAA, VEGF-1 and VEGF-2 may also be a result of the stress response, which counteracts the effects of mundoserone on angiogenesis. Thus, mundoserone and other anti-VEGF drugs should be combined for anti-angiogenesis treatment (36). Fifth, other angiogenesis-associated mechanisms should be examined in the future, including matrix metalloproteinases $(37,38)$.

In conclusion, the present study identified a novel compound, mundoserone, which may be an effective angiogenic inhibitor, which acts via downregulation of the SLIT/ROBO1 and FGFR/PTP-RB pathways, as well as upregulation of NOTCH1A signaling (Fig. 4). Further in vitro studies are necessary to confirm the role of mundoserone on the proliferation and migration of microvascular endothelial cells via the above signaling pathways.

\section{Acknowledgements}

Not applicable.

\section{Funding}

The present study was supported by National Natural Science Foundation of China (grant no. 81500331) and the Science and Technology Commission of Shanghai (grant nos. 17140902600 and 14JC1404400).

\section{Availability of data and materials}

The datasets used and/or analyzed during the current study are available from the corresponding author on reasonable request.

\section{Authors' contributions}

$\mathrm{KC}, \mathrm{CW}$ and $\mathrm{YF}$ designed the study and interpreted the data. $\mathrm{JG}, \mathrm{ZH}$ and $\mathrm{YW}$ collected the data. LG and $\mathrm{HZ}$ performed the statistical analyses. $\mathrm{KC}, \mathrm{JG}$ and $\mathrm{ZH}$ prepared the figures. All authors wrote the manuscript. All authors read and approved the final version of the manuscript.

\section{Ethical approval and consent to participate}

This study was approved by the Institutional Animal Care and Use Committee of Shanghai Ninth People's Hospital.

\section{Patient consent for publication}

Not applicable.

\section{Competing interests}

The authors declare that they have no competing interests.

\section{References}

1. Zhao J, Zhang ZR, Zhao N, Ma BA and Fan QY: VEGF silencing inhibits human osteosarcoma angiogenesis and promotes cell apoptosis via PI3K/AKT signaling pathway. Int J Clin Exp Med 8: 12411-12417, 2015.

2. Duan J, Hu H, Feng L, Yang X and Sun Z: Silica nanoparticles inhibit macrophage activity and angiogenesis via VEGFR2-mediated MAPK signaling pathway in zebrafish embryos. Chemosphere 183: 483-490, 2017.

3. Kim BS, Park JY, Kang HJ, Kim HJ and Lee J: Fucoidan/FGF-2 induces angiogenesis through JNK- and p38-mediated activation of AKT/MMP-2 signalling. Biochem Biophys Res Commun 450: 1333-1338, 2014.

4. Hasan SS, Tsaryk R, Lange M, Wisniewski L, Moore JC, Lawson ND, Wojciechowska K, Schnittler H and Siekmann AF: Endothelial Notch signalling limits angiogenesis via control of artery formation. Nat Cell Biol 19: 928-940, 2017.

5. Xu H, Zhang Y, Peña MM, Pirisi L and Creek KE: Six1 promotes colorectal cancer growth and metastasis by stimulating angiogenesis and recruiting tumor-associated macrophages. Carcinogenesis 38: 281-292, 2017.

6. Malecic N and Young HS: Excessive angiogenesis associated with psoriasis as a cause for cardiovascular ischaemia. Exp Dermatol 26: 299-304, 2017.

7. Lu Y, Yu SS, Zong M, Fan SS, Lu TB, Gong RH, Sun LS and Fan LY: Glucose-6-phosphate isomerase (G6PI) mediates hypoxia-induced angiogenesis in rheumatoid arthritis. Sci Rep 7: 40274, 2017.

8. Abu El-Asrar AM, Struyf S, Mohammad G, Gouwy M, Rytinx P, Siddiquei MM, Hernández C, Alam K, Mousa A, De Hertogh $G$, et al: Osteoprotegerin is a new regulator of inflammation and angiogenesis in proliferative diabetic retinopathy. Invest Ophthalmol Vis Sci 58: 3189-3201, 2017.

9. Rakhila H, Al-Akoum M, Bergeron ME, Leboeuf M, Lemyre M, Akoum A and Pouliot M: Promotion of angiogenesis and proliferation cytokines patterns in peritoneal fluid from women with endometriosis. J Reprod Immunol 116: 1-6, 2016.

10. OH WK, McDermott D, Porta C, Levy A, Elaidi R, Scotte F Hawkins R, Castellano D, Bellmunt J, Rha SY, et al: Angiogenesis inhibitor therapies for advanced renal cell carcinoma: Toxicity and treatment patterns in clinical practice from a global medical chart review. Int J Oncol 44: 5-16, 2014. 
11. Motzer RJ, Porta C, Vogelzang NJ, Sternberg CN, Szczylik C, Zolnierek J, Kollmannsberger C, Rha SY, Bjarnason GA, Melichar B, et al: Dovitinib versus sorafenib for third-line targeted treatment of patients with metastatic renal cell carcinoma: An open-label, randomised phase 3 trial. Lancet Oncol 15: 286-296, 2014

12. Patridge E, Gareiss P, Kinch MS and Hoyer D: An analysis of FDA-approved drugs: Natural products and their derivatives. Drug Discov Today 21: 204-207, 2016.

13. Rathinasamy VS, Paneerselvan N and Ragunathan M: Effect of genistein on regenerative angiogenesis using zebrafish as model organism. Biomed Prev Nutri 4: 469-474, 2014.

14. Song CX, Song SL, Liang H and Liu X: Effect of camptothecin on the embryonic development and angiogenesis of zebrafish embryos. Adv Mater Res 750-752: 1472-1475, 2013.

15. Liang F, Han Y, Gao H, Xin S, Chen S, Wang N, Wei Q, Zhong H, Lin S, Yao X and Li S: Kaempferol identified by zebrafish assay and fine fractionations strategy from dysosma versipellis inhibits angiogenesis through VEGF and FGF pathways. Sci Rep 5: 14468,2015

16. Yang GW, Jiang JS and Lu WQ: Ferulic acid exerts anti-angiogenic and anti-tumor activity by targeting fibroblast growth factor receptor 1-mediated angiogenesis. Int J Mol Sci 16: 24011-24031, 2015.

17. Zhao D, Qin C, Fan X, Li Y and Gu B: Inhibitory effects of quercetin on angiogenesis in larval zebrafish and human umbilical vein endothelial cells. Eur J Pharmacol 723: 360-367, 2014.

18. Pantel J, Williams SY, Mi D, Sebag J, Corbin JD, Weaver CD and Cone RD: Development of a high throughput screen for allosteric modulators of melanocortin-4 receptor signaling using a real time cAMP assay. Eur J Pharmacol 660: 139-147, 2011.

19. Cross LM, Cook MA, Lin S, Chen JN and Rubinstein AL: Rapid analysis of angiogenesis drugs in a live fluorescent zebrafish assay. Arterioscler Thromb Vasc Biol 23: 911-912, 2003.

20. Pakes SP: Adequate veterinary care as viewed by the American Association for Accreditation of Laboratory Animal Care. J Am Vet Med Assoc 168: 519-521, 1976.

21. Livak KJ and Schmittgen TD: Analysis of relative gene expression data using real-time quantitative PCR and the 2(-Delta Delta C(T)) method. Methods 25: 402-408, 2001

22. Yeh JR, Munson KM, Elagib KE, Goldfarb AN, Sweetser DA and Peterson RT: Discovering chemical modifiers of oncogene-regulated hematopoietic differentiation. Nat Chem Biol 5: 236-243, 2009

23. Srivastava $P$ and Panda D: Rotenone inhibits mammalian cell proliferation by inhibiting microtubule assembly through tubulin binding. FEBS J 274: 4788-4801, 2007.

24. Ishido M and Suzuki J: Inhibition by rotenone of mesencephalic neural stem-cell migration in a neurosphere assay in vitro. Toxicol In Vitro 24: 552-557, 2010.

25. Zhang L, Yao K, Fan Y, He P, Wang X, Hu W and Chen Z: Carnosine protects brain microvascular endothelial cells against rotenone-induced oxidative stress injury through histamine $\mathrm{H}_{1}$ and $\mathrm{H}_{2}$ receptors in vitro. Clin Exp Pharmacol Physiol 39: 1019-1025, 2012.

26. Hatanaka K, Lanahan AA, Murakami M and Simons M: Fibroblast growth factor signaling potentiates VE-cadherin stability at adherens junctions by regulating SHP2. PLoS One 7: e37600, 2012.
27. Sauteur L, Krudewig A, Herwig L, Ehrenfeuchter N, Lenard A, Affolter $\mathrm{M}$ and Belting HG: Cdh5/VE-cadherin promotes endothelial cell interface elongation via cortical actin polymerization during angiogenic sprouting. Cell Rep 9: 504-513, 2014.

28. Wang B, Xiao Y, Ding BB, Zhang N, Yuan Xb, Gui L, Qian KX, Duan S, Chen Z, Rao Y and Geng JG: Induction of tumor angiogenesis by Slit-Robo signaling and inhibition of cancer growth by blocking Robo activity. Cancer Cell 4: 19-29, 2003.

29. Fujiwara M, Ghazizadeh M and Kawanami O: Potential role of the Slit/Robo signal pathway in angiogenesis. Vasc Med 11: $115-121,2006$

30. Yang XM, Han HX, Sui F, Dai YM, Chen M and Geng JG: Slit-Robo signaling mediates lymphangiogenesis and promotes tumor lymphatic metastasis. Biochem Biophys Res Commun 396: 571-577, 2010.

31. Liao WX, Laurent LC, Agent S, Hodges J and Chen DB: Human placental expression of SLIT/ROBO signaling cues: Effects of preeclampsia and hypoxia. Biol Reprod 86: 111, 2012.

32. Rama N, Dubrac A, Mathivet T, Ní Chárthaigh RA, Genet G, Cristofaro B, Pibouin-Fragner L, Ma L, Eichmann A and Chédotal A: Slit2 signaling through Robo1 and Robo2 is required for retinal neovascularization. Nat Med 21: 483-491, 2015.

33. Banerjee D, Hernandez SL, Garcia A, Kangsamaksin T, Sbiroli E, Andrews J, Forrester LA, Wei N, Kadenhechiweshe A, Shawber CJ, et al: Notch suppresses angiogenesis and progression of hepatic metastases. Cancer Res 75: 1592-1602, 2015.

34. Lee SH, Lee S, Yang H, Song S, Kim K, Saunders TL, Yoon JK, Koh GY and Kim I: Notch pathway targets proangiogenic regulator Sox 17 to restrict angiogenesis. Circ Res 115: 215-226, 2014.

35. Liu J, Deutsch U, Jeong J and Lobe CG: Constitutive notch signaling in adult transgenic mice inhibits bFGF-induced angiogenesis and blocks ovarian follicle development. Genesis 52: 809-816, 2015.

36. Butler CT, Reynolds AL, Tosetto M, Dillon ET, Guiry PJ, Cagney G, O'Sullivan J and Kennedy BN: A quininib analogue and cysteinyl leukotriene receptor antagonist inhibits vascular endothelial growth factor (VEGF)-independent Angiogenesis and exerts an additive antiangiogenic response with bevacizumab. J Biol Chem 292: 3552-3567, 2017.

37. Webb AH, Gao BT, Goldsmith ZK, Irvine AS, Saleh N, Lee RP, Lendermon JB, Bheemreddy R, Zhang Q, Brennan RC, et al: Inhibition of MMP-2 and MMP-9 decreases cellular migration, and angiogenesis in in vitro models of retinoblastoma. BMC Cancer 17: 434, 2017.

38. Wang J, Chen D, Li B, He J, Duan D, Shao D and Nie M: Fe-MIL-101 exhibits selective cytotoxicity and inhibition of angiogenesis in ovarian cancer cells via downregulation of MMP. Sci Rep 26: 26126, 2016.

This work is licensed under a Creative Commons Attribution-NonCommercial-NoDerivatives 4.0 International (CC BY-NC-ND 4.0) License. 